\title{
ON PROJECTION CONSTANT PROBLEMS AND THE EXISTENCE OF METRIC PROJECTIONS IN NORMED SPACES
}

\author{
E. M. EL-SHOBAKY, SAHAR MOHAMMED ALI, \\ AND WATARU TAKAHASHI
}

Received 3 September 2001

We give the sufficient conditions for the existence of a metric projection onto convex closed subsets of normed linear spaces which are reduced conditions than that in the case of reflexive Banach spaces and we find a general formula for the projections onto the maximal proper subspaces of the classical Banach spaces $l_{p}, 1 \leq p<\infty$ and $c_{0}$. We also give the sufficient and necessary conditions for an infinite matrix to represent a projection operator from $l_{p}, 1 \leq p<\infty$ or $c_{0}$ onto anyone of their maximal proper subspaces.

\section{Introduction}

A subset $C$ of a normed linear space $X$ is called an existence subset of $X$ if and only if for every element $x \in X$ there is an element $y \in C$, where $y$ is called the best approximation of $x$ denoted by $b(x, C)[2,3]$ such that

$$
\|x-y\|=\operatorname{dist}(x, C):=\inf \{\|x-y\|: y \in C\} .
$$

Needless to say, best approximations play a major role in many applications, including approximation theory, optimization and applications in mathematical economics and engineering. Thus, the mathematical analysis of the properties of the best approximation elements has drawn much attention in research.

In $[11,13]$, the authors there used the terms Čebyšev subsets and proximal subsets instead of the existence subsets and studied the characterizations of the existence subsets of Banach spaces.

It is shown that if $X$ is a reflexive Banach space and $C$ is a closed convex subset, then for every $x \in X$ the best approximation element $b(x, C)$ exists and is unique. 
402 Projection constant problems and metric projections

In this paper, reduced assumptions on a normed linear space for a closed convex subset to exist are given, instead of the reflexivity and the completeness assumptions of the given normed space.

On the other hand, it is known that the existence of a projection $P$ from a Banach space $X$ onto its closed subspace $Y$ is equivalent to the existence of an extension $\widehat{T}$ of any operator $T$ from $Y$ into $W$ to an operator from $X$ into $W$ such that $\|\widehat{T}\| \leq\|P\|\|T\|$. The two equivalent problems, that is

(1) how small can the norm of the extended operator be made? and

(2) what is the projection of smallest norm?,

are challenging to the study of the relative projection constant $\lambda(Y, X)$ of $Y$ in $X$, where

$$
\lambda(Y, X):=\inf \{\|P\|, P \text { is a projection from } X \text { onto } Y\}
$$

and the absolute projection constant of $Y, \lambda(Y)$, where

$$
\lambda(Y):=\sup \{\lambda(Y, X), X \text { contains } Y \text { as a closed subspace }\} \text {. }
$$

In [10], the upper estimate for the absolute projection constant $\lambda(Y)$ of a finitedimensional space $Y$ with $\operatorname{dim} Y=n$ is found in the form

$$
\lambda(Y) \leq \begin{cases}\sqrt{n}-\frac{1}{\sqrt{n}}+O\left(n^{-3 / 4}\right), & \text { in the real field } \\ \sqrt{n}-\frac{1}{2 \sqrt{n}}+O\left(n^{-3 / 4}\right), & \text { in the complex field }\end{cases}
$$

The precise values for $l_{1}^{n}, l_{2}^{n}$, and $l_{n}^{p}, p \neq 1, p \neq 2$, have been calculated by Grünbaum [8], Gordon [7], Garling and Gordon [6], Rutovitz [12], and König et al. [9].

In [5], interesting results have been given for the injective and projective tensor products.

For a finite codimensional subspaces, Garling and Gordon [6] showed that if $Y$ is a finite codimensional subspace of the space $X$ with co-dimension $n$, then for every $\epsilon>0$ there exists a projection $P$ from $X$ onto $Y$ with norm

$$
\|P\| \leq 1+(1+\epsilon) \sqrt{n} .
$$

And so, $\lambda(Y, X) \leq 1+\sqrt{n}$. In particular, if the co-dimension of $Y$ is 1 , that is, $Y$ is a hyperplane in the space $X$, then $\lambda(Y, X) \leq 2$.

\section{Notation and basic definitions}

We will use the same notation that is given in $[1,4,14]$. 
Let $C$ be a nonempty closed convex subset of a normed space $X$. If for every $x \in X$ there is a unique $b(x, C)$ in $C$, then the mapping $b(x, C)$ is said to be a metric projection onto $C$, in this case we have

$$
\|x-b(x, C)\|=\operatorname{dist}(x, C) \quad \forall x \in X .
$$

Clearly, if $X$ is a Hilbert space and $C$ is a nonempty closed convex subset of $X$, then there is a metric projection from $X$ onto $C$, see [14].

As a direct consequence of the separation theorem, if $X$ is a locally convex linear topological space, then a nonempty convex subset $C$ of $X$ is closed in the strong topology of $X$ if and only if $C$ is closed in the weak topology of $X$, see [4].

A relative projection constant of the closed subspace $Y$ in the space $X$ is said to be exact if and only if there is a projection $P$ from $X$ onto $Y$ at which the infimum of (1.2) is attained.

A subspace $Y$ of the space $X$ is said to be a hyperplane (maximal proper subspace) of the space $X$ if and only if $X$ contains $Y$ as a subspace of deficiency 1 .

It is known that a subspace $Y$ is a hyperplane of the space $X$ if and only if there is a functional $f \in X^{*}$ such that $Y=f^{-1}(\{0\})$; see [1].

Let $P$ be an operator on the space $X$. Then the point $x \in X$ is said to be a maximal point of the operator $P$ if and only if $\|P\|=\|P(x)\|$.

If $X$ is either the Banach space $l_{\infty}$, the Banach space of all bounded scalarvalued functions $\left\{x_{n}\right\}_{n=1}^{\infty}$ on a countably infinite set $N$, or $l_{p}$ the Banach space of all scalar-valued functions $x=\left\{x_{n}\right\}_{n=1}^{\infty}$ on a countably infinite set $N$, such that $\sum_{n=1}^{\infty}\left|x_{n}\right|^{p}<\infty$ or $c_{0}$ the closed subspace of the Banach space $l_{\infty}$, then the norms on $X$ are defined as follows:

$$
\|x\|_{X}:= \begin{cases}\sup _{n=1}^{\infty}\left|x_{n}\right| & \text { if } X=l_{\infty}, \\ \left(\sum_{n=1}^{\infty}\left|x_{n}\right|^{p}\right)^{1 / p} & \text { if } X=l_{p} .\end{cases}
$$

Our first result is the following theorem.

THEOREM 2.1. Let $X$ be a normed space in which every Cauchy sequence has a weakly convergent subsequence and the parallelogram law holds. Let $C$ be a nonempty closed convex subset of $X$. Then the metric projection $b(\cdot, C)$ from $X$ onto $C$ exists.

Proof. Let $x \in X$ and consider the distance function $\operatorname{dist}(x, C)$, there exists a sequence $\left\{y_{n}\right\}_{n=1}^{\infty}$ of elements in $C$ such that

$$
\operatorname{dist}(x, C) \leq\left\|x-y_{n}\right\| \leq \operatorname{dist}(x, C)+\frac{1}{n}, \quad n=1,2, \ldots
$$


Taking the limit as $n \rightarrow \infty$, we get $\lim _{n \rightarrow \infty}\left\|x-y_{n}\right\|=\operatorname{dist}(x, C)$. The sequence $\left\{y_{n}\right\}_{n=1}^{\infty}$ is a Cauchy sequence in $X$. In fact, using the convexity of $C$, we have $(1 / 2)\left(y_{i}+y_{j}\right) \in C$, also using the parallelogram law, we have

$$
\left\|\left(y_{i}-x\right)-\left(y_{j}-x\right)\right\|^{2}+\left\|\left(y_{i}-x\right)+\left(y_{j}-x\right)\right\|^{2}=2\left\|y_{i}-x\right\|^{2}+2\left\|y_{j}-x\right\|^{2} .
$$

Therefore,

$$
\begin{aligned}
\left\|y_{i}-y_{j}\right\|^{2}= & 2\left\|y_{i}-x\right\|^{2}+2\left\|y_{j}-x\right\|^{2}-4\left\|x-\frac{1}{2}\left(y_{i}+y_{j}\right)\right\|^{2} \\
\leq & 2\left[\operatorname{dist}(x, C)+\frac{1}{i}\right]^{2}+2\left[\operatorname{dist}(x, C)+\frac{1}{j}\right]^{2} \\
& -4 \operatorname{dist}(x, C)^{2} \stackrel{i, j \rightarrow \infty}{\longrightarrow} 0,
\end{aligned}
$$

using the assumption, every Cauchy sequence has a weakly convergent subsequence, the sequence $\left\{y_{n}\right\}_{n=1}^{\infty}$ has a subsequence $\left\{y_{n_{i}}\right\}_{i=1}^{\infty}$ converging weakly to some point $y_{0}$ in $X, y_{n_{i}} \underset{\text { weakly }}{\stackrel{i \rightarrow \infty}{\longrightarrow}} y_{0}$. Since $C$ is convex and is closed in the strong topology, $C$ is closed in the weak topology, then $C$ contains as well all its weak limits $y_{0} \in C$. Now, consider the proper convex lower semi-continuous real-valued function $g$ on $C$ defined by

$$
g(z)=\|x-z\| \quad \forall z \in C,
$$

we have

$$
\lim _{n \rightarrow \infty} g\left(y_{n}\right)=\lim _{n \rightarrow \infty}\left\|x-y_{n}\right\|=\operatorname{dist}(x, C),
$$

and the mapping $g$ attains its minimum at $y_{0}$. In fact, let $\epsilon>0$. Then the set

$$
G_{g\left(y_{0}\right)-\epsilon}=\left\{z \in C: g(z) \leq g\left(y_{0}\right)-\epsilon\right\}
$$

is a convex closed subset of $X$. Using the convexity of $G_{g\left(y_{0}\right)-\epsilon}$, for every $\epsilon>0$ the set $G_{g\left(y_{0}\right)-\epsilon}$ is a weakly closed subset of $C$ and hence for every $\epsilon>0$ the set

$$
G_{g\left(y_{0}\right)-\epsilon}^{c(C)}=\left\{z \in C: g(z)>g\left(y_{0}\right)-\epsilon\right\}
$$

is a weakly open subset of $C$, since $y_{0} \in G_{g\left(y_{0}\right)-\epsilon}^{c(C)}$, there is a neighborhood $V$ of $y_{0}$ (w.r.t. the weak topology) such that $V \subset G_{g\left(y_{0}\right)-\epsilon}^{c(C)}$. Using the weak limit point definition of the sequence $\left\{y_{n_{i}}\right\}_{i=1}^{\infty}$, there is $i_{0} \in N$ such that $y_{n_{i}} \in V$ for all $i \geq i_{0}$, then $y_{n_{i}} \in G_{g\left(y_{0}\right)-\epsilon}^{c(C)}$ for all $i \geq i_{0}$. Therefore $g\left(y_{n_{i}}\right)>g\left(y_{0}\right)-\epsilon$ for every $i \geq i_{0}$. Finally we have 
E. M. El-Shobaky et al. 405

$$
\begin{aligned}
\inf \{g(z): z \in C\} & \leq g\left(y_{0}\right) \leq \inf _{i \geq i_{0}} g\left(y_{n_{i}}\right)+\epsilon \leq \liminf _{i} g\left(y_{n_{i}}\right)+\epsilon \\
& =\lim _{i \rightarrow \infty} g\left(y_{n_{i}}\right)+\epsilon=\lim _{n \rightarrow \infty} g\left(y_{n}\right)+\epsilon \\
& =\inf \{g(z): z \in C\}+\epsilon .
\end{aligned}
$$

Since $\epsilon>0$ is arbitrary and $y_{0} \in C$, we have

$$
g\left(y_{0}\right)=\min \{g(z): z \in C\} .
$$

Thus for every $x \in X$ there is $y_{0} \in C$ such that

$$
\left\|x-y_{0}\right\|=\min \{\|x-z\|: z \in C\}=\operatorname{dist}(x, C) .
$$

To show that such a point $y_{0}$ is unique, let $g\left(y_{0}\right)=0$. Since $\left\|x-y_{0}\right\|=0$, $x=y_{0}, y_{0}$ is unique. Let $g\left(y_{0}\right)>0$ and let $z_{0}$ be an element in $C$ with

$$
g\left(z_{0}\right)=\left\|x-z_{0}\right\|=\min \{\|x-z\|: z \in C\}=g\left(y_{0}\right)=\left\|x-y_{0}\right\|
$$

and $z_{0} \neq y_{0}\left(\left\|z_{0}-y_{0}\right\|>0\right)$, since $C$ is convex, $(1 / 2)\left(y_{0}+z_{0}\right) \in C$, using the parallelogram law, we have

$$
\begin{aligned}
g\left(y_{0}\right) & \leq g\left(\frac{1}{2}\left(y_{0}+z_{0}\right)\right)=\left\|x-\frac{1}{2}\left(y_{0}+z_{0}\right)\right\| \\
& =\frac{1}{2}\left\|\left(x-y_{0}\right)+\left(x-z_{0}\right)\right\|=\frac{1}{2}\left(\left\|\left(x-y_{0}\right)+\left(x-z_{0}\right)\right\|^{2}\right)^{1 / 2} \\
& =\frac{1}{2}\left(2\left\|\left(x-y_{0}\right)\right\|^{2}+2\left\|\left(x-z_{0}\right)\right\|^{2}-\left\|z_{0}-y_{0}\right\|^{2}\right)^{1 / 2} \\
& <\frac{1}{2}\left(2\left\|\left(x-y_{0}\right)\right\|^{2}+2\left\|\left(x-z_{0}\right)\right\|^{2}\right)^{1 / 2} \\
& =\frac{1}{2}\left(2 g\left(y_{0}\right)^{2}+2 g\left(z_{0}\right)^{2}\right)^{1 / 2}=g\left(y_{0}\right) .
\end{aligned}
$$

This is a contradiction. Therefore no such $z_{0}$ exists, and $y_{0}$ is unique. Now, define the mapping $b(x, C)$ from $X$ onto $C$ by $b(x, C)=y_{0}$, the mapping $b(x, C)$ is the required metric projection.

Our result concerning the maximal proper subspaces of the classical Banach spaces $c_{0}$ or $l_{p}$ for $1 \leq p<\infty$ is the following result.

TheOREM 2.2. Let $X$ denote one of the spaces $c_{0}$ or $l_{p}$ for $1 \leq p<\infty, f \in X^{*}$ and $Y$ the closed linear subspace $Y=f^{-1}(\{0\})=\left\{y=\left\{b_{i}\right\}_{i=1}^{\infty}: f(y)=\right.$ $\left.\sum_{i=1}^{\infty} b_{i} f_{i}=0\right\}$ of the space $X$. Then the general formula of any projection from $X$ onto $Y$ is given by

$$
P=I_{l_{p}}-f \otimes z \quad \text { for some } z \in X \text { with } f(z)=1 \text {. }
$$


406 Projection constant problems and metric projections

Proof. Since every element $x=\left\{x_{i}\right\}_{i=1}^{\infty} \in X$ is uniquely written as $x=$ $\sum_{i=1}^{\infty} x_{i} e_{i}$, any operator $P$ on $X$ is completely determined by $P\left(e_{i}\right)$, suppose that $P\left(e_{i}\right)=\left\{e_{i k}\right\}_{k=1}^{\infty}$, we have

$$
P(x)=\sum_{i \geq 1} x_{i} P\left(e_{i}\right)=\left\{\sum_{i \geq 1} x_{i} e_{i k}\right\}_{k=1}^{\infty} .
$$

Define the element $z=\left\{\alpha_{k}\right\}_{k=1}^{\infty} \in X$ as follows

$$
f_{i} \alpha_{k}=\delta_{i k}-e_{i k}, \quad k \geq 1
$$

Since $f$ is a nonzero element of the space $l_{1}$ or $l_{q}$, where $1 / p+1 / q=1, f \neq 0$, there is at least one index $i$ for which $f_{i} \neq 0$, for this index multiplying (2.17) by $f_{k}$ and summing with respect to $k$, we get

$$
f_{i} \sum_{k \geq 1} f_{k} \alpha_{k}=\sum_{k \geq 1} f_{k}\left(\delta_{i k}-e_{i k}\right)=f_{i}-\sum_{k \geq 1} f_{k} e_{i k}
$$

Since $P\left(e_{i}\right) \in Y$ and $f\left(P\left(e_{i}\right)\right)=0$, that is, $\sum_{k \geq 1} f_{k} e_{i k}=0$. Therefore, $f_{i} \sum_{k \geq 1} f_{k} \alpha_{k}=f_{i}$, this proves that $\sum_{k \geq 1} f_{k} \alpha_{k}=1$, that is, $f(z)=1$. On the other hand, we have $e_{i k}=\delta_{i k}-\alpha_{k} f_{i}$. Thus the representation of $P$ is as follows:

$$
\begin{aligned}
P(x) & =\left\{\sum_{i \geq 1} x_{i}\left(\delta_{i k}-\alpha_{k} f_{i}\right)\right\}_{k=1}^{\infty}=\left\{x_{k}-\alpha_{k}\left(\sum_{i \geq} x_{i} f_{i}\right)\right\}_{k=1}^{\infty} \\
& =\left\{x_{k}-\alpha_{k} f(x)\right\}_{k=1}^{\infty}=x-f(x)\left\{\alpha_{k}\right\}_{k=1}^{\infty}=x-f(x) z .
\end{aligned}
$$

The converse direction is clearly true. To calculate the norm of the given projection $P$, we have two distinct cases, the first is when $X=c_{0}$ in which the norm is as follows:

$$
\begin{aligned}
\|P\| & =\sup _{\|x\|=1}\left\|\left\{\sum_{i=1}^{\infty} \delta_{i k} x_{i}-\alpha_{k} \sum_{i \geq 1} x_{i} f_{i}\right\}_{k=1}^{n}\right\|_{l_{\infty}} \\
& =\sup _{k \geq 1}\left(\left|1-\alpha_{k} f_{k}\right|+\left|\alpha_{k}\right|\left[\|f\|_{l_{1}}-\left|f_{k}\right|\right]\right) .
\end{aligned}
$$


The second case is when $X=l_{p}$ in which the norm of $P$ is as follows:

$$
\begin{aligned}
\|P\| & =\sup _{\|x\|=1}\left\|\left\{\sum_{i \geq 1} \delta_{i k} a_{i}-\alpha_{k} \sum_{i \geq 1} x_{i} f_{i}\right\}_{k=1}^{\infty}\right\|_{l_{p}} \\
& =\sup _{\|x\|=1}\left\|\left\{\sum_{i \geq 1}\left(\delta_{i k}-\alpha_{k} f_{i}\right) x_{i}\right\}_{k=1}^{\infty}\right\|_{l_{p}} \\
& =\sup _{\|x\|=1}\left\|\left\{\left\{\delta_{i k}-\alpha_{k} f_{i}\right\}_{i=1}^{\infty}\left(\left\{x_{i}\right\}_{i=1}^{\infty}\right)\right\}_{k=1}^{\infty}\right\|_{l_{p}} \\
& =\sup _{\|x\|=1}\left(\sum_{k \geq 1}\left|\left\{\delta_{i k}-\alpha_{k} f_{i}\right\}_{i=1}^{\infty}\left(\left\{x_{i}\right\}_{i=1}^{\infty}\right)\right|^{p}\right)^{1 / p} \\
& =\left(\sum_{k \geq 1} \sup _{\|x\|=1}\left|\left\{\delta_{i k}-\alpha_{k} f_{i}\right\}_{i=1}^{\infty}\left(\left\{x_{i}\right\}_{i=1}^{\infty}\right)\right|^{p}\right)^{1 / p} \\
& =\left(\sum_{k \geq 1}\left(\left\|\left\{\delta_{i k}-\alpha_{k} f_{i}\right\}_{i=1}^{\infty}\right\|_{l_{q}}\right)^{p}\right)^{1 / p}=\left(\sum_{k \geq 1}\left(\sum_{i=1}^{\infty}\left|\delta_{i k}-\alpha_{k} f_{i}\right|^{q}\right)^{p / q}\right)^{1 / p} .
\end{aligned}
$$

Corollary 2.3. If $f=\left\{f_{i}\right\}_{i=1}^{\infty}$ is an element of the space $\left(l_{1}\right)^{*}=l_{\infty}$ and $Y$ is a subspace of the space $l_{1}, Y=f^{-1}(\{0\})$, then some of the maximal points of any projection $P$ from $l_{1}$ onto $Y$ lie in the set $\left\{e_{i}\right\}_{i=1}^{\infty}$, where $\left\{e_{i}\right\}_{i=1}^{\infty}$ is the canonical basis of $l_{1}$.

Proof. According to Theorem 2.2, the norm of any projection in this case is given by

$$
\begin{aligned}
\|P\| & =\sup _{\|x\|=1}\|p(x)\|=\sup _{\|x\|=1}\left\|x-f(x) z_{0}\right\| \\
& =\sup _{\|x\|=1}\left\|\left\{\sum_{i \geq 1} \delta_{i k} x_{i}-\alpha_{k} \sum_{i \geq 1} x_{i} f_{i}\right\}_{k=1}^{\infty}\right\|_{l_{1}} \\
& \leq \sum_{k \geq 1} \sup _{\|x\|=1}\left|\left\{\delta_{i k}-\alpha_{k} f_{i}\right\}_{i=1}^{\infty}\left(\left\{x_{i}\right\}_{i=1}^{\infty}\right)\right| \\
& =\sum_{k \geq 1}\left\|\left\{\delta_{i k}-\alpha_{k} f_{i}\right\}_{i=1}^{\infty}\right\|_{l_{\infty}}=\sum_{k \geq 1} \sup _{i \geq 1}\left|\delta_{i k}-\alpha_{k} f_{i}\right| \\
& =\sup _{i \geq 1} \sum_{k \geq 1}\left|\delta_{i k}-\alpha_{k} f_{i}\right|=\sup _{i \geq 1}\left\|P\left(e_{i}\right)\right\| .
\end{aligned}
$$


Therefore the norm of any such projection is attained at some points in the set $\left\{e_{i}\right\}_{i=1}^{\infty}$.

Remark 2.4. If one of the coordinates of $f$, say $f_{i}=0$, then $e_{i} \in Y$ (for $f\left(e_{i}\right)=$ $f_{i}=0$ ). And to get a norm one projection the effect of this projection on the basis elements must not exceed one.

Corollary 2.5. Let $X$ denote one of the spaces $c_{0}$ or $l_{p}, 1<p<\infty$, and let $f \in X^{*}$. Then an infinite matrix $P=\left\{p_{i n}\right\}_{i, n \in N}$ represents a projection operator from $X$ onto its hyperplane $f^{-1}(\{0\})$ if and only if the matrix $\alpha=$ $\left\{\delta_{i n}-p_{i n}\right\}_{i, n \in N}$ satisfies the following conditions:

(1) trace $\alpha=1$,

(2) each row of the matrix $\alpha$ is a scalar multiple of $f$.

Proof. Let $P=\left\{p_{i n}\right\}_{i, n \in N}$ be an infinite matrix satisfying conditions (1) and (2). Then $P=I-\alpha$, since each row of the matrix $\alpha$ is a scalar multiple of $f$, $\alpha(y)=0$ for all $y \in f^{-1}(\{0\})$. And so, $P(y)=y$ for all $y \in f^{-1}(\{0\})$. If $f^{n}$ denotes the $n$th row of the matrix $\alpha$, using condition (2) we obtain for each $n \in N$ a scalar $\alpha_{n}$ such that $f^{n}=\alpha_{n} f$, using condition (1) we have $f(z)=1$, where $z=\left\{\alpha_{n}\right\}_{n=1}^{\infty}$. To show that the range of $P$ is $f^{-1}(\{0\})$ it is sufficient to show that $f(P(x))=0$ for all $x=\left\{x_{i}\right\}_{i=1}^{\infty} \in X$, so suppose that $x=\left\{x_{i}\right\}_{i=1}^{\infty} \in X$, we have

$$
\begin{aligned}
f(P(x)) & =f(I-\alpha)(x)=f(x)-f(\alpha(x))=f(x)-f\left(\left\{\alpha_{n} f_{i}\right\}_{i, n \in N}(x)\right) \\
& =f(x)-f\left(\left\{\sum_{i=1}^{\infty} \alpha_{n} f_{i} x_{i}\right\}_{n \in N}\right)=f(x)-\sum_{n=1}^{\infty} f_{n} \sum_{i=1}^{\infty} \alpha_{n} f_{i} x_{i} \\
& =f(x)-\sum_{n=1}^{\infty} f_{n} \alpha_{n} \times \sum_{i=1}^{\infty} f_{i} x_{i}=f(x)-f(z) f(x)=0 .
\end{aligned}
$$

Conversely, if $P$ is a projection from $X$ onto $f^{-1}(\{0\})$, using Theorem 2.2, we obtain an element $z=\left\{\alpha_{n}\right\}_{n=1}^{\infty} \in X$ such that $f(z)=1$ and $P=I_{X}-f \otimes z$. So

$$
\begin{aligned}
P\left(\left\{x_{i}\right\}_{i=1}^{\infty}\right) & =I_{X}(x)-f\left(\left\{x_{i}\right\}_{i=1}^{\infty}\right) \times z=I_{X}(x)-\sum_{i=1}^{\infty} f_{i} x_{i} \times z \\
& =I_{X}(x)-\left\{z_{n} \sum_{i=1}^{\infty} f_{i} x_{i}\right\}_{n=1}^{\infty}=\left\{\delta_{i n}-\alpha_{i n}\right\}_{i, n \in N}\left(\left\{x_{i}\right\}_{i=1}^{\infty}\right)
\end{aligned}
$$

where $\alpha_{i n}=z_{i} f_{n}$. This proves that the operator $P$ has the matrix representation $P=\left\{\delta_{i n}-\alpha_{i n}\right\}_{i, n \in N}$. Clearly the matrix $\alpha=\left\{\alpha_{i n}\right\}_{i, n \in N}$ satisfies conditions (1) and (2). 
CoROLlary 2.6. Let $f=\delta\left\{\epsilon_{k}\right\}_{k=1}^{n}, n>2$, be a sequence of the space $l_{n}^{1}$, where $\delta$ is a nonzero scalar and $\epsilon_{i}= \pm 1$. Then the relative projection constant of the $(n-1)$-dimensional subspace $f^{-1}(\{0\})$ in the space $l_{n}^{\infty}$ is given by

$$
\lambda\left(f^{-1}(\{0\}), l_{n}^{\infty}\right)=2-\frac{2}{n} .
$$

Moreover, the minimal norm projection is given by

$$
P_{0}(x)=x-\frac{f(x)}{\|f\|_{l_{n}^{2}}^{2}} \times f .
$$

Proof. As given in Theorem 2.2 the norm of any projection corresponding to the element $z=\left\{\alpha_{k}\right\}_{k=1}^{n}$ is given by

$$
\begin{aligned}
\|P\| & =\sup _{k=1}^{n}\left(\left|1-\alpha_{k} f_{k}\right|+\left|\alpha_{k}\right|\left[\|f\|_{l_{n}^{1}}-\left|f_{k}\right|\right]\right) \\
& =\sup _{k=1}^{n}\left(\left|1-\delta \alpha_{k} \epsilon_{k}\right|+\left|\alpha_{k}\right|[n-1]|\delta|\right) .
\end{aligned}
$$

Assume that the minimal projection is a norm one projection. Then there is $z \in l_{n}^{1}$ and

$$
\left|1-\delta \alpha_{k} \epsilon_{k}\right|+[n-1]\left|\alpha_{k}\right||\delta| \leq 1,
$$

for every $k=1,2, \ldots, n$. In this case, we have $1-\left|\delta \alpha_{k}\right|+[n-1]\left|\alpha_{k}\right||\delta| \leq 1$ for every $k=1,2, \ldots, n$. Therefore $[n-2]|\delta| \alpha_{k} \mid \leq 0$ for every $k=1,2, \ldots, n$. For $n>2$, this is true only if $\alpha_{k}=0$ for every $k=1,2, \ldots, n$. This is an obvious contradiction, thus there is no norm one projection from $l_{n}^{1}$ onto $f^{-1}(\{0\})$.

Now, let $x=\left\{x_{k}\right\}_{k=1}^{n} \in l_{n}^{\infty}$ be an arbitrary point. To project this point to the point $x_{0}=\left\{x_{k}^{0}\right\}_{k=1}^{n}$ in the space $f^{-1}(\{0\})$ with a minimal available distance between the points $x=\left\{x_{k}\right\}_{k=1}^{n}$ and $x_{0}=\left\{x_{k}^{0}\right\}_{k=1}^{n}$, the sequence $x_{0}-x=$ $\left\{x_{k}^{0}-x_{k}\right\}_{k=1}^{n}$ must be parallel to the line passing through $f=\left\{f_{k}\right\}_{k=1}^{n}$ and perpendicular to the plane $f^{-1}(\{0\})$. Thus there is a scalar $\lambda$ such that $x_{0}-x=$ $\lambda f$. On the other hand, since $x_{0} \in f^{-1}(\{0\}), f\left(x_{0}\right)=0$, thus $0=f\left(x_{0}\right)=$ $f(x)+\lambda\|f\|_{l_{n}^{2}}^{2}$ and so $\lambda=-f(x) /\|f\|_{l_{n}^{2}}^{2}$, it follows that $x_{0}=x-f(x) /\|f\|_{l_{n}^{2}}^{2} \times$ $f$. The required projection $P_{0}$ from $l_{n}^{\infty}$ onto $f^{-1}(\{0\})$ is defined by the formula

$$
P_{0}\left(x=\left\{x_{k}\right\}_{k=1}^{n}\right)=x_{0}=x-\frac{f(x)}{\|f\|_{l_{n}^{2}}^{2}} \times f .
$$

(Note that the element $z_{0}$ corresponding to $P_{0}$ is $z_{0}=f /\|f\|_{l_{n}^{2}}^{2}$ and also $\left\|P_{0}\right\|=$ $(2-2 / n)$.

Now we are going to show that this projection is a minimal norm projection. Assume the contrary, that is, there exists an element $z \in l_{n}^{1}$ such that $f(z)=1$ 
410 Projection constant problems and metric projections

and the corresponding projection $P$ satisfies $\|P\|<(2-2 / n)$, according to (2.27), we have

$$
\left(\left|1-\delta \alpha_{k} \epsilon_{k}\right|+\left|\alpha_{k}\right|[n-1]|\delta|\right)<\left(2-\frac{2}{n}\right)
$$

for every $k \in\{1,2, \ldots, n\}$, from which we get

$$
[n-2]\left|\alpha_{k}\right||\delta|<\left(1-\frac{2}{n}\right)
$$

and so for such $z$ we have

$$
\left|\alpha_{k}\right|<\frac{1}{n|\delta|} \quad \forall k=1,2, \ldots, n,
$$

multiplying by $\left|f_{k}\right|=|\delta|$, summing with respect to $k$, we get $\sum_{k=1}^{n}\left|f_{k}\right|\left|\alpha_{k}\right|<1$. On the other hand, the inequality

$$
1=\sum_{k=1}^{n} f_{k} \alpha_{k} \leq \sum_{k=1}^{n}\left|f_{k}\right|\left|\alpha_{k}\right|<1
$$

gives a contradiction, hence no such $z$ exists, from which we concluded the proof.

Corollary 2.7. If $f=\left\{f_{n}\right\}_{n=1}^{\infty}$ is a sequence of the space $l_{1}$, and $f^{-1}(\{0\})$. Then $\lambda\left(f^{-1}(\{0\}), c_{0}\right)=1$ if and only if there is $n \in N$ for which $\left|f_{n}\right| \geq$ $(1 / 2)\|f\|_{l_{1}}$.

Proof. As given in Theorem 2.2, the norm of any projection corresponding to the element $z$ is given by

$$
\|P\|=\sup _{k \geq 1}\left(\left|1-\alpha_{k} f_{k}\right|+\left|\alpha_{k}\right|\left[\|f\|_{l_{1}}-\left|f_{k}\right|\right]\right) .
$$

To have a norm one projection, we must have $\left|1-\alpha_{k} f_{k}\right|+\left|\alpha_{k}\right|\left(\|f\|_{l_{1}}-\left|f_{k}\right|\right) \leq 1$ for every $k \in N$. In this case we have $1-\left|\alpha_{k} f_{k}\right|+\left|\alpha_{k}\right|\left(\|f\|_{l_{1}}-\left|f_{k}\right|\right) \leq 1$ for every $k \in N$. Therefore

$$
\left|\alpha_{k}\right|\left(\|f\|_{l_{1}}-2\left|f_{k}\right|\right) \leq 0 \quad \forall k \in N .
$$

If $k \in N$ and $\left(\|f\|_{l_{1}}-2\left|f_{k}\right|\right)>0$, then $\left|\alpha_{k}\right|=0$, but $f\left(z_{0}\right)=1$ implies that at least one $k \in N$ for which $\left|\alpha_{k}\right| \neq 0$ and so at least one $k \in N$ for which $\left(\|f\|_{l_{1}}-\right.$ $\left.2\left|f_{k}\right|\right) \leq 0$. Therefore there is at least $k \in N$ for which $\left|f_{k}\right| \geq(1 / 2)\|f\|_{l_{1}}$.

EXAMPLE 2.8. The minimal norm projection of the subspace $Y=\left\{x=\left\{b_{i}\right\}_{i=1}^{3} \mid\right.$ $\left.\sum_{i=1}^{3} b_{i}=0\right\}$ in the space $l_{3}^{\infty}$ is the projection given by

$$
P_{0}\left(\left\{x_{i}\right\}_{i=1}^{3}\right)=\frac{1}{3}\left\{2 x_{1}-x_{2}-x_{3}, 2 x_{2}-x_{1}-x_{3}, 2 x_{3}-x_{1}-x_{2}\right\} \text {. }
$$

with norm $\left\|P_{0}\right\|=4 / 3$. 


\section{References}

[1] M. M. Day, Normed Linear Spaces, Ergebnisse der Mathematik und ihrer Grenzgebiete. Neue Folge. Heft, vol. 21, Springer-Verlag, Berlin, 1958. MR 20:1187. Zbl 0082.10603 .

[2] F. S. De Blasi and J. Myjak, On a generalized best approximation problem, J. Approx. Theory 94 (1998), no. 1, 54-72. MR 99i:41034. Zbl 0912.41015.

[3] F. S. De Blasi, J. Myjak, and P. L. Papini, Porous sets in best approximation theory, J. London Math. Soc. (2) 44 (1991), no. 1, 135-142. MR 92h:41066. Zbl 0786.41027.

[4] N. Dunford and J. T. Schwartz, Linear Operators. I. General Theory, Pure and Applied Mathematics, vol. 6, Interscience, New York, 1958. MR 22:8302. Zbl 0084.10402.

[5] E. M. El-Shobaky, S. M. Ali, and W. Takahashi, On the projection constants of some topological spaces and some applications, Abstr. Appl. Anal. 6 (2001), no. 5, 299-308.

[6] D. J. H. Garling and Y. Gordon, Relations between some constants associated with finite dimensional Banach spaces, Israel J. Math. 9 (1971), 346-361. MR 54:896. Zbl 0212.14203.

[7] Y. Gordon, On the projection and Macphail constants of $l_{p}^{n}$ spaces, Israel J. Math. 6 (1968), 295-302. MR 38:4961. Zbl 0182.45202.

[8] B. Grünbaum, Projection constants, Trans. Amer. Math. Soc. 95 (1960), 451-465. MR 22:4937. Zbl 0095.09002.

[9] H. König, C. Schütt, and N. Tomczak-Jaegermann, Projection constants of symmetric spaces and variants of Khintchine's inequality, J. Reine Angew. Math. 511 (1999), 1-42. MR 2000i:46014. Zbl 0926.46008.

[10] H. König and N. Tomczak-Jaegermann, Norms of minimal projections, J. Funct. Anal. 119 (1994), no. 2, 253-280. MR 94m:46024. Zbl 0818.46015.

[11] R. R. Phelps, Čebyšev subspaces of finite codimension in $C(X)$, Pacific J. Math. 13 (1963), 647-655. MR 27:6069. Zbl 0115.10101.

[12] D. Rutovitz, Some parameters associated with finite-dimensional Banach spaces, J. London Math. Soc. 40 (1965), 241-255. MR 32:8120. Zbl 0125.06402.

[13] I. Singer, Best Approximation in Normed Linear Spaces by Elements of Linear Subspaces, Die Grundlehren der mathematischen Wissenschaften, vol. 171, SpringerVerlag, New York, 1970. MR 42:4937. Zbl 0197.38601.

[14] W. Takahashi, Nonlinear Functional Analysis, Fixed Point Theory and Its Applications, Yokohama Publishers, Yokohama, 2000.

E. M. El-Shobaky: Department of Mathematics, Faculty of Science, Ain Shams UNIVERSITY, CAIRo, EGYPT

E-mail address: solar@ photoenergy.org

Sahar Mohammed Ali: Department of Mathematics, Faculty of Science, Ain Shams University, CAIro, Egypt

E-mail address: sahar0@is.titech.ac.jp

Wataru Takahashi: Department of Mathematical and Computing Sciences, Tokyo Institute of Technology, 2-12-1 Ookayama, Meguro-ku, Tokyo 152-8552, JAPAN

E-mail address: wataru@is.titech.ac.jp 


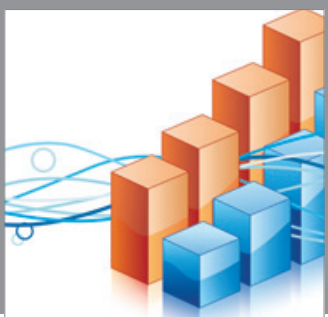

Advances in

Operations Research

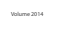

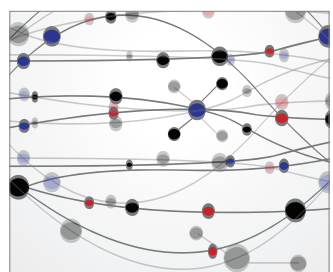

\section{The Scientific} World Journal
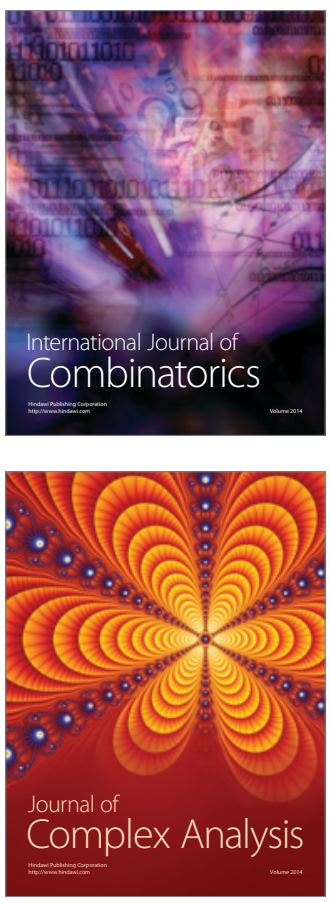

International Journal of

Mathematics and

Mathematical

Sciences
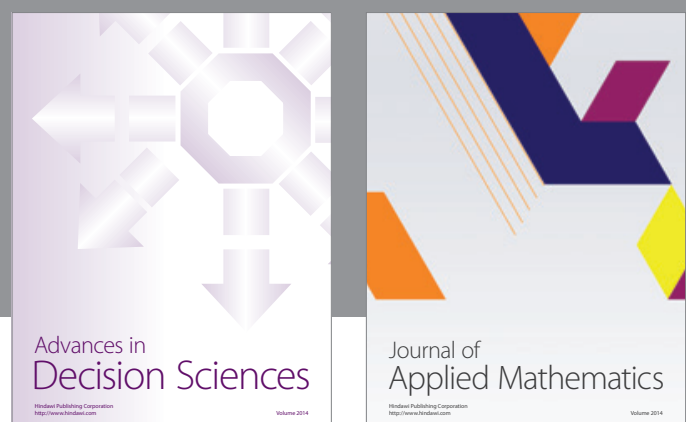

Journal of

Applied Mathematics
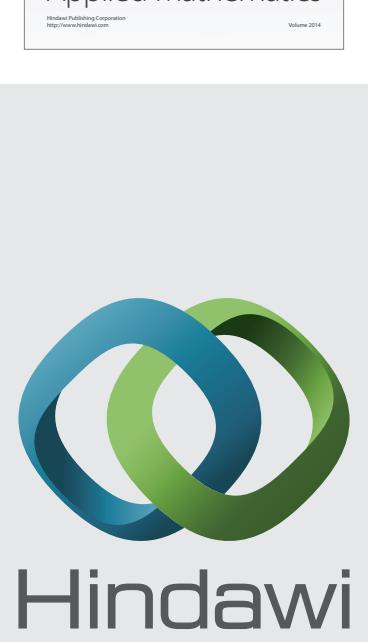

Submit your manuscripts at http://www.hindawi.com
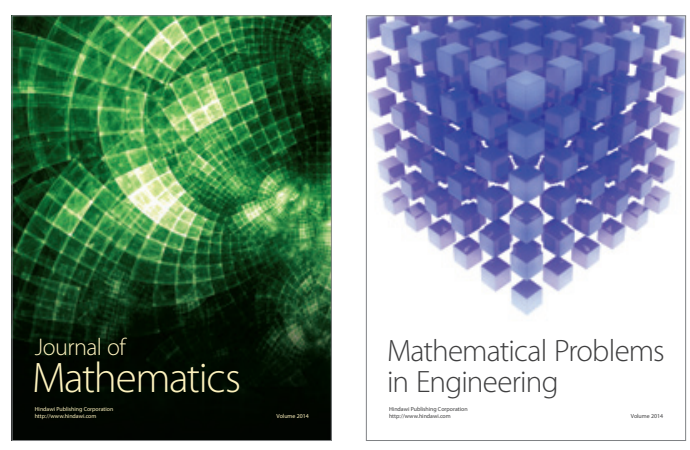

Mathematical Problems in Engineering
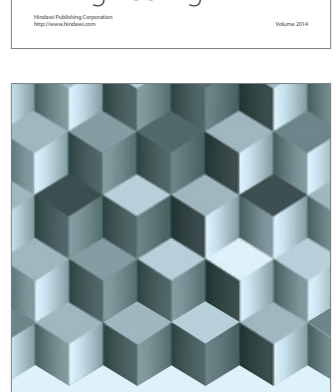

Journal of

Function Spaces
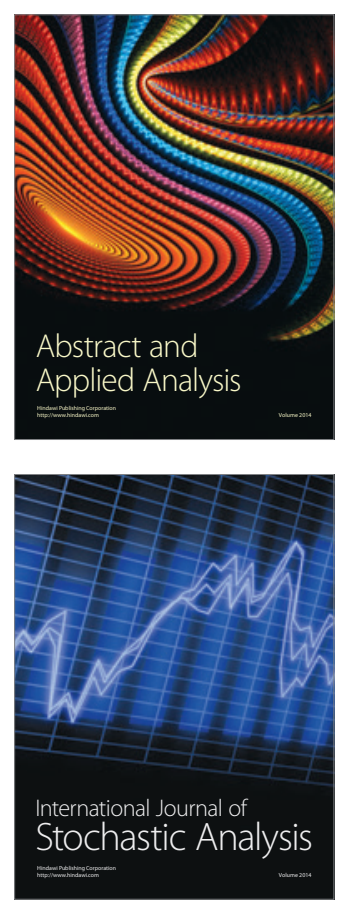

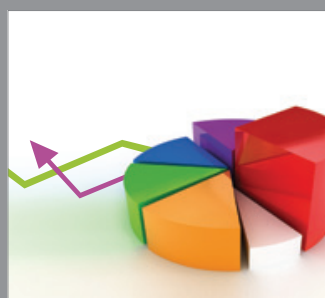

ournal of

Probability and Statistics

Promensencen
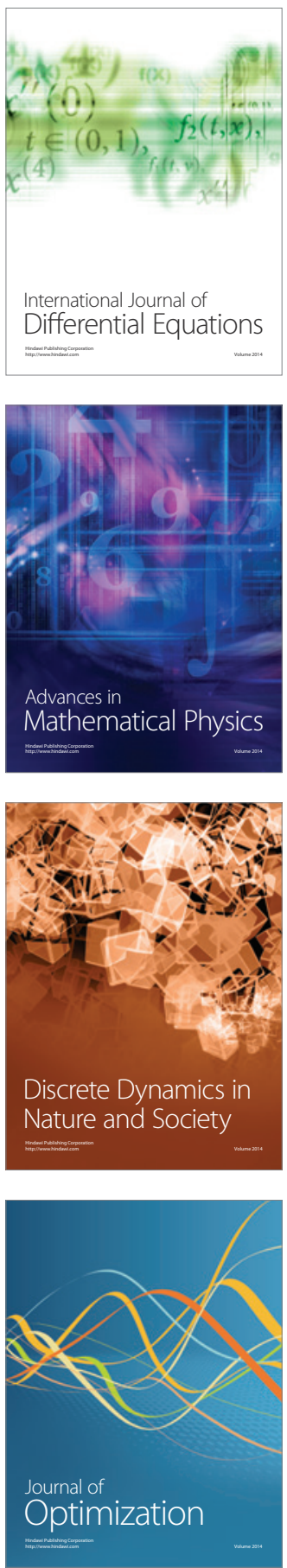